\title{
QUELQUES REMARQUES \\ CONCERNANT LA FORMATION \\ DE LA MATIÈRE GRASSE PAR LE PORC
}

PAR

André-M. LEROY

Professeur de Zootechnie à l'Institut National Agronomique

\section{PLAN DU MÉMOIRE}

I. - Importance et signification des quotients respiratoires supérieurs à l'unité constatés chez les porcs normalement alimentés.

11. - Analyse et utilisation des données d'un récent travail de J. Ludvigsen et $G$. Thorbek concernant l'effet $d$ 'un antibiotique sur le métabolisme du porc.

III. - Mécanismes de la consommation d'oxygène et du rejet de gaz carbonique par les porcs, entre 20 et 100 kilogrammes.

IV. - Mise en évidence d'importantes variations individuelles chez des porcs soumis au même régime alimentaire.

v. - Influence de la formation de la graisse sur le coefficient permettant de calculer l'ensemble des pertes de chaleur provenant des actions dynamiques spécifiques engendrées par la consommation des aliments.

VI. - Influence de la formation de la graisse sur le rapport entre le poids vif gagné par l'animal et sa consommation alimentaire évaluée en unités fourragères.

VII. - Conclusions pratiques résultant des observations formulées dans le cours du mémoire.

\section{PREMIÈRE, PAR'TIE}

Importance et signification des quotients respiratoires supérieurs à l'unité constatés chez les pores normalement alimentés.

Dans le cours de la seconde moitié du xix ${ }^{\mathrm{e}}$ siècle, de nombreux auteurs ont démontré que les animaux pouvaient transformer en matière 
grasse les excédents de matériaux hydrocarbonés alimentaires qui n'étaient pas utilisés aux besoins de la calorification.

Des preuves évidentes de cette transformation ont été apportées presque au même moment par Soxhl,ET, MUssL et STrohmer, TCHIRWinsky, Voit et LEHMANn et RUbNer. I a formule brutale de cette transformation, qui ne préjuge en rien des mécanismes complexes et délicats exigés par cette dernière, et qui n'a d'autre prétention, pour cette raison, que de rendre compte du point de départ et du point d'arrivée des éléments mis en jeu, a été indiquée pour la première fois par Armand GaUtier, puis confirmée par RICHET et HANRIOT. Elle possède la formule suivante:

$$
\underset{\text { Glucose }}{\mathrm{I}_{3} \mathrm{C}_{6} \mathrm{H}_{12} \mathrm{O}_{6}}=\underset{\text { Oleostearopalmitine }}{\mathrm{C}_{55} \mathrm{O}_{104} \mathrm{O}_{6}}+23 \mathrm{CO}_{2}+26 \mathrm{H}_{2} \mathrm{O}
$$

La transformation, probablement exothermique, n'exige pas la présence de l'oxygène, et postule un important dégagement de gaz carbonique.

Les expériences effectuées sur les porcs par FingERLING, par MOLLGAARD et par nous-même, avec la collaboration de ZELTER, ont nettement mis en évidence l'existence chez le porc de quotients respiratoires apparents supérieurs à l'unité, pouvant s'élever parfois jusqu'à I, 4, selon l'éloignement des repas. Ces coefficients ont tendance à augmenter à mesure que le porc avance en âge, ce qui est en parfait accord avec l'accroissement correspondant de la quantité de graisse quotidiennement formée par les animaux. Il nous a paru intéressant d'étudier de près ce phénomène, afin de voir dans quelle mesure il serait possible d'en tirer parti pour mettre en évidence l'existence, sur les animaux en cours d'engraissement, de différences individuelles. Nous avions également l'intention, en entreprenant le présent travail, d'examiner dans quelle mesure la relation d'A. GAUTIER pouvait permettre d'expliquer les résultats expérimentaux constatés par les auteurs précités.

\section{DEUXIÈME: PARTIE}

\section{Analyse et utilisation des données d'un récent travail de J. Ludvigsen et G. Thorbek, concernant l'efiet d'un antibiotique sur le métabolisme du porc.}

Deux élèves de MoligaArd, J. Ludvigsen et G. Thorbek, ont publié en I955, dans la collection des C. R. des travaux des stations expérimentales du Danemark ( $\mathrm{N}^{\circ}$ 283), un fort intéressant travail sur les échanges nutritifs et respiratoires de porcs normalement alimentés. Ces animaux ont été divisés en deux lots composés chacun de 4 mâles et de 4 femelles ; ils recevaient la même alimentation, complétée seulement pour le lot expérimental $n^{\circ} 2$ par une addition d'auréomycine, à la dose 
quotidienne de 20 milligrammes. Chaque porc a été examiné à six reprises, en chambre respiratoire, pendant un temps suffisant pour qu'il soit possible d'étudier ses échanges de matières et d'énergie par l'emploi combiné des deux méthodes classiques de calorimétrie indirecte. Les résultats de chaque expérience, qui figurent in extenso dans le mémoire, ont été publiés dans la forme suivante, devenue classique à la suite des travaux de KELINER.

\section{TABleaU I}

Mode d'expression des résultats observés par LUDVIGSEN et THORBEK au cours de leurs expériences sur la détermination des échanges nutritifs des porcs.

Femelle $n^{\circ}$ I : Première période d'observation, 2 et 9 Mars 1954 . - Régime alimentaire prévu pour des porcs entre 20 et $25 \mathrm{~kg}$. - Balance de l'azote, du carbone et de l'énergie.

\begin{tabular}{|c|c|c|c|c|c|}
\hline & $\begin{array}{l}\text { Masse } \\
\text { recueillie } \\
\longrightarrow\end{array}$ & $\begin{array}{c}\text { Matière } \\
\text { sèche } \\
-\end{array}$ & $\begin{array}{l}\text { Azote } \\
\text { total } \\
-\end{array}$ & Carbone & Calories \\
\hline Aliments..... & $213^{8} \mathrm{~g}$ & $843,8 \mathrm{~g}$ & $3^{2,43} \mathrm{~g}$ & $384, \mathrm{I} \mathrm{g}$ & 3713 \\
\hline Fèces........ & 6 го $\mathrm{g}$ & I 39,9 g & $5,55 \mathrm{~g}$ & $6 \mathrm{r}, 7 \mathrm{~g}$ & $63^{6}$ \\
\hline Urine........ & I 3 r $3 \mathrm{~g}$ & & $\mathrm{I} \mathrm{I}, 07 \mathrm{~g}$ & I I,9 g & I I5 \\
\hline $\begin{array}{l}\text { Retenu ...... } \\
\text { Rejeté...... }\end{array}$ & & $703,9 \mathrm{~g}$ & $\begin{array}{l}26,88 \mathrm{~g} \\
\mathrm{r} 5,8 \mathrm{r} \mathrm{g}\end{array}$ & & \\
\hline
\end{tabular}

Échanges respiratoires

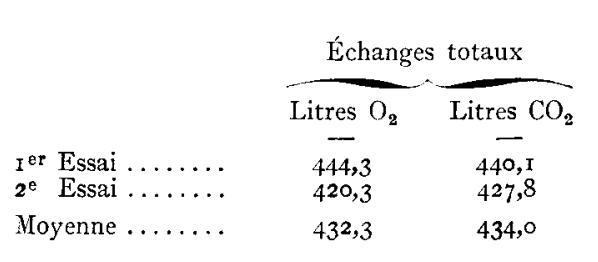

Balance du carbone

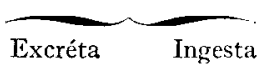

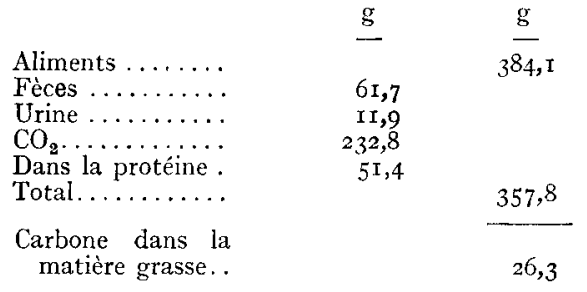

Ces $26,3 \mathrm{~g}$ de carbone correspondent à $34,4 \mathrm{~g}$ de graisse contenant 325 calories

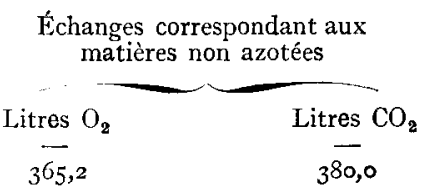

Quotient respiratoire correspondant : $\mathbf{I} \mathbf{0 4} \mathbf{I}$

Balance de l'énergie

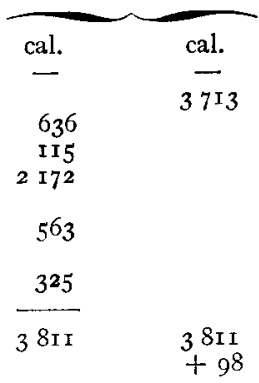

Différence........

Erreur expérimentale 2,6\%
Production de chaleur évaluée par calorimétrie indirecte..... Détermination de la graisse produite, après correction corres-

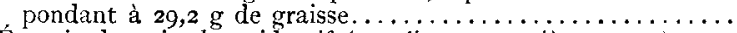
Énergie du gain de poids vif (protéine + matière grasse)....

$$
\begin{aligned}
2 \text { 1 } 72-49 & =2 \text { I23 calories } \\
325-49 & =276 \text { calories } \\
563+276 & =839 \text { calories }
\end{aligned}
$$

D'après le plan d'alimentation publié par les auteurs, il a été possible de calculer, pour chacune des expériences, les quantités de ma- 
tières hydrocarbonées et de matières grasses digestibles utilisées par chaque animal. D'autre part, les données du mémoire correspondant à chaque expérience nous ont permis de déduire de la quantité totale de chaleur perdue la fraction de cette quantité correspondant aux matières azotées désassimilées et d'obtenir ainsi la part de la dépense énergétique provenant des matériaux non azotés.

L'énergie correspondant aux matières azotées désassimilées se calcule en effet de la manière suivante, indiquée par les auteurs du mémoire à la page 24 de ce dernier :

Énergie calorifique provenant des matières azotées $=\mathrm{N}$ urinaire $\times 6,25 \times 4,5$ calories. Ainsi, par exemple, pour l'expérience dont les détails figurent ci-dessus, la quantité de chaleur empruntée aux matériaux non azotés de la ration consommée s'obtient par les opérations suivantes :

Dépense métabolique totale mesurée par calorimétrie indirecte..

$\mathrm{N}$ urinaire : $\mathrm{I} \mathrm{I}, \mathrm{O} 7$

Fraction de la dépense métabolique correspondant aux matières

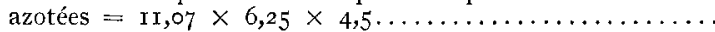

Fraction de la dépense métabolique correspondant aux matières

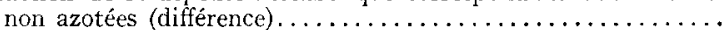

2123 calories

3 I calories

I8I 2 calories

$\mathrm{Si}$ nous supposons que la totalité de cette dépense métabolique correspondant aux matières non azotées provient uniquement des glucides disparus au cours des processus métaboliques, le résultat que nous obtiendrons en retranchant de l'énergie correspondant aux matières hydrocarbonées digérées la fraction de l'énergie métabolisée correspondant à ces mêmes matières nous fournira la quantité de glucides digestibles disponibles pour être transformés en graisse. Or, d'après la relation d'Armand GAUTIER, un gramme de matière hydrocarbonée peut donner au cours de cette transformation $0,3675 \mathrm{~g}$ de matière grasse. Nous pouvons donc ainsi connaître dans chaque cas la quantité de matière grasse correspondant aux glucides qui ne sont pas utilisés à des fins de calorification.

Il est légitime d'admettre, d'autre part, que la totalité des matières grasses peuvent se déposer après de faibles remaniements moléculaires ne modifiant pas leur masse dans les réserves adipeuses de chaque sujet. $\mathrm{Si}$ nous admettons cette hypothèse, il devient possible de calculer dans chaque cas la quantité de matière grasse qui peut enrichir quotidiennement le corps de l'animal, et de comparer le résultat obtenu à la détermination expérimentale correspondante.

Ainsi, par exemple, pour l'expérience dont nous avons donné précédemment les détails, la quantité de matière hydrocarbonée digérée est de $380 \mathrm{~g}$, correspondant à I 920 calories. La fraction de cette quantité d'énergie transformable en graisse est de:

$$
\text { I } 920-\mathrm{I} 8 \mathrm{I} 2=\mathrm{I} 08 \text { calories }
$$




\section{TABLEAU II}

Comparaison des quantités de matière grasse prévues par le calcul et des quantités correspondantes mesurées expérimentalement.

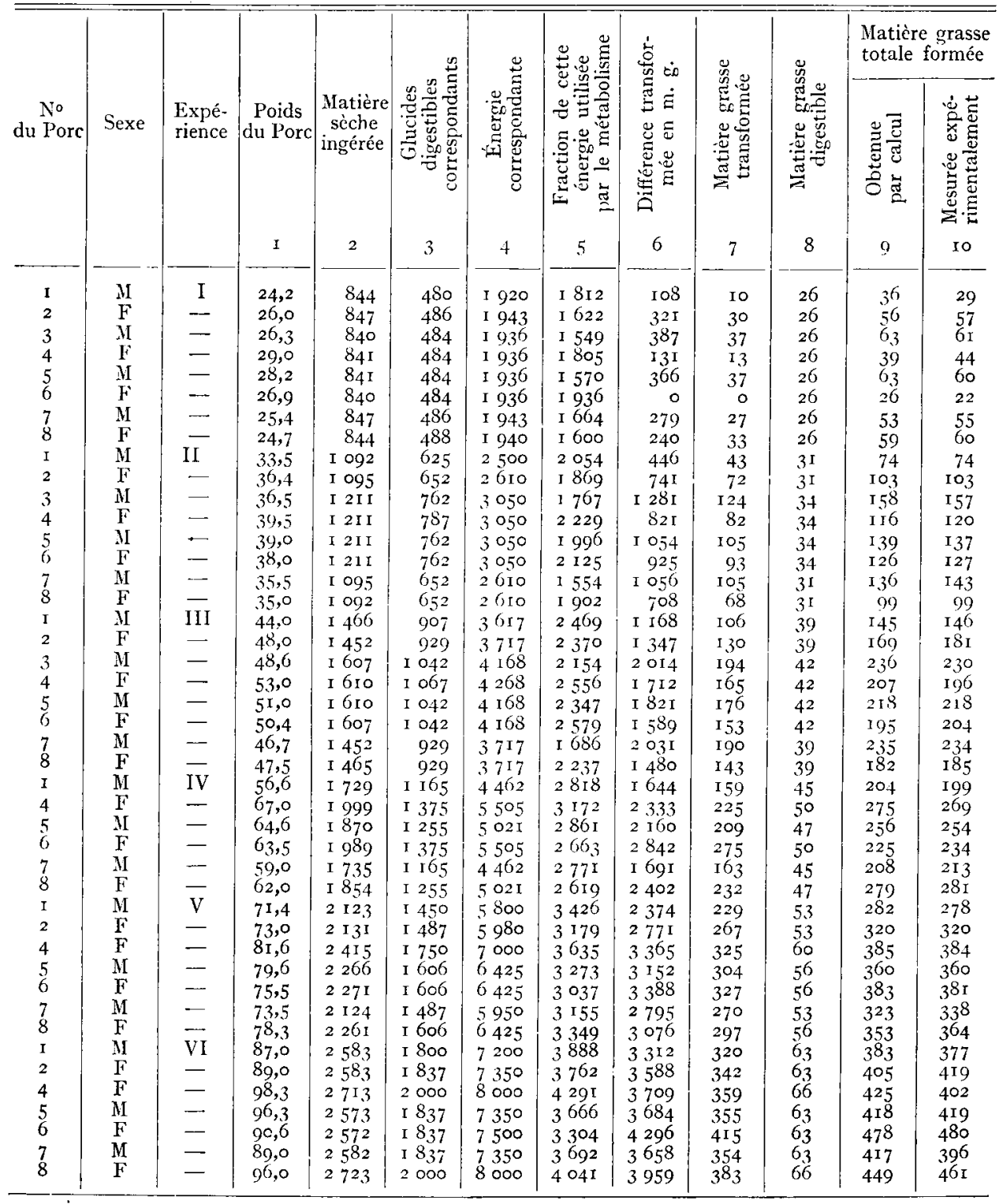

La confrontation des résultats qui figurent dans les deux colonnes de droite du tableau, montre avec évidence que les quantités de matière grasse obtenues par les deux méthodes sont, dans le plus grand nombre des cas, exactement superposables, ce qui est une excellente preuve de la valeur de la formule d'A. Gautier. 
ce qui correspond à $28,4 \mathrm{~g}$ de glucose, dont la transformation en graisse fournit $28,4 \times 0,3675=10,4 \mathrm{~g}$ de cette dernière.

D'autre part, les aliments consommés par l'animal lui apportent $25,8 \mathrm{~g}$ de matière grasse digestible. La quantité de graisse de dépôt fournie par notre calcul Io,4 $+25,8=36,2 \mathrm{~g}$, peut ainsi se comparer à la quantité correspondante obtenue par l'expérience, qui est de $26,2 \mathrm{~g}$.

Dans le tableau II, nous avons fait figurer tous les calculs analogues, pour les 8 sujets et les 6 séries expérimentales. Seul, le sujet $\mathrm{n}^{\mathrm{o}} 3$ ne figure que dans les 3 premières séries.

En valeur absolue, l'erreur moyenne entre les deux déterminations de la matière grasse, obtenues, la première par calcul et la deuxième, expérimentalement, qui est de 2,36 , est nettement inférieure à celle que les auteurs danois ont trouvée entre les résultats des deux méthodes de calorimétrie utilisées par eux, laquelle s'élève à 7,6 I (pour 44 couples de données expérimentales) ${ }^{1}$ ).

\section{TROISIÈME PARTIE}

\section{Hécanismes de la consommation d'oxygène et du rejet de gaz carbonique par les pores, entre 20 et 100 kilogrammes.}

D'après l'hypothèse précédemment admise, la totalité des matières non azotées utilisées à la calorification est fournie par la fraction des glucides digestibles qui n'est pas transformée en graisse. Puisque nous connaissons la quantité d'énergie provenant de l'utilisation de ces glucides, qui figure dans la colonne 5 du tableau II, il est facile d'en déduire la quantité de glucose provenant de ces derniers et correspondant à chaque cas. Ce nombre est égal à celui de la colonne 4 divisé par la valeur calorifique d'un gramme de glucide, que nous avons fixée à 3,9 calories par gramme.

D'après l'équation théorique de la combustion du glucose :

$$
\mathrm{C}_{6} \mathrm{H}_{12} \mathrm{O}_{6}+6 \mathrm{O}_{2}=6 \mathrm{CO}_{2}+6 \mathrm{H}_{2} \mathrm{O}
$$

Une quantité de $180 \mathrm{~g}$ de glucose s'unit à $192 \mathrm{~g}$ d'oxygène et fournit $264 \mathrm{~g}$ de gaz carbonique accompagné de $108 \mathrm{~g}$ d'eau. Un gramme de glucose utilisé à des fins énergétiques exige donc $\mathrm{I}, 067 \mathrm{~g}$ d'oxygène et correspond à une émission de $1,467 \mathrm{~g}$ de gaz carbonique. Transformés en volume à 0 et $760 \mathrm{~mm}$, les quantités correspondantes de gaz sont de 0,751 pour l'oxygène comme pour le gaz carbonique.

Nous pouvons ainsi calculer les quantités d'oxygène nécessaires à la combustion totale des glucides disparus au cours de chaque expérience, et les comparer aux quantités correspondantes mesurées expérimenta-

(1) Pour l'expérience I effectuée avec la femelle $n^{0}$ I dont nous avons donné les résultats détaillés, cette erreur est de $34,4-29,2=6,2$. Entre les données correspondantes du tableau II, l'écart est de 7 . 
lement, qui figurent dans le mémoire de Ludvigsen et Thorbek. Ainsi, pour l'expérience de la sétie I, effectuée avec la femelle No I, le nombre

\section{TABLEAU III}

Résultats fournis par les calculs et par les mesures de l'oxygène absorbé et du gaz carbonique, émis au cours de chaque expérience.

\begin{tabular}{|c|c|c|c|c|c|c|c|c|c|c|}
\hline \multirow{3}{*}{$\begin{array}{l}\text { No du } \\
\text { Porc }\end{array}$} & \multirow{3}{*}{$\left|\begin{array}{c}\text { Série } \\
\text { expéri- } \\
\text { mentale }\end{array}\right|$} & \multicolumn{4}{|c|}{$\begin{array}{c}\text { Données concernant l'oxygène } \\
\text { absorbé }\end{array}$} & \multicolumn{5}{|c|}{ Données concernant le gaz carbonique émis } \\
\hline & & \multirow[t]{2}{*}{$\begin{array}{c}\mathrm{O}_{2} \\
\text { absorbe } \\
\text { total }\end{array}$} & \multirow{2}{*}{$=\begin{array}{c}\mathrm{O}_{2} \text { corres- } \\
\text { pondant } \\
\text { aux mat. } \\
\text { azotées }\end{array}$} & \multicolumn{2}{|c|}{$\begin{array}{c}\mathrm{O}_{2} \text { correspondant } \\
\text { aux matières } \\
\text { non azotées } \mathrm{O}_{2} \\
\end{array}$} & \multirow{2}{*}{$\begin{array}{l}\mathrm{CO}_{2} \\
\text { émis. } \\
\text { total }\end{array}$} & \multirow{2}{*}{$\begin{array}{l}\mathrm{CO}_{2} \text { corr. } \\
\text { aux mat. } \\
\text { azotées }\end{array}$} & \multirow{2}{*}{$\mid \begin{array}{c}\mathrm{CO}_{2} \text { corr. } \\
\text { aux mat. } \\
\text { non } \\
\text { azotées }\end{array}$} & \multicolumn{2}{|c|}{$\begin{array}{l}\mathrm{CO}_{2} \text { excédentaire } \\
\text { prov.de la forma- } \\
\text { tion de la graisse }\end{array}$} \\
\hline & & & & Calculé & Mesuré & & & & Calculé & Mesuré \\
\hline & & $\begin{array}{l}\mathrm{I} \\
\mathrm{g}\end{array}$ & $\begin{array}{l}\mathrm{I} \\
2\end{array}$ & $\begin{array}{l}\mathrm{I} \\
3\end{array}$ & $\begin{array}{l}\mathrm{T} \\
4\end{array}$ & $\begin{array}{l}1 \\
5\end{array}$ & $\begin{array}{l}\text { I } \\
6\end{array}$ & $\begin{array}{l}\mathrm{I} \\
7\end{array}$ & $\begin{array}{l}\mathbf{I} \\
8\end{array}$ & $\begin{array}{l}\text { I } \\
9\end{array}$ \\
\hline $\begin{array}{l}\mathrm{I} \\
2\end{array}$ & I & $\begin{array}{r}432 \\
387\end{array}$ & 67 & 348 & $3^{65}$ & 434 & 54 & 380 & 6 & I 5 \\
\hline $\begin{array}{l}2 \\
3\end{array}$ & 二 & $\begin{array}{l}387 \\
403\end{array}$ & 55 & $3 \mathrm{I} 2$ & 332 & 388 & 45 & 343 & 18 & II \\
\hline 4 & - & 428 & $\begin{array}{r}90 \\
123\end{array}$ & $\begin{array}{l}297 \\
346\end{array}$ & $\begin{array}{l}3 \mathrm{I} 3 \\
370\end{array}$ & 410 & 72 & 338 & 22 & 25 \\
\hline 5 & 一 & 400 & 86 & 302 & $\begin{array}{l}370 \\
3 \mathbf{I} 4\end{array}$ & $\begin{array}{l}42 \mathrm{I} \\
409\end{array}$ & $\begin{array}{l}99 \\
70\end{array}$ & $\begin{array}{l}375 \\
339\end{array}$ & $\begin{array}{r}8 \\
22\end{array}$ & 5 \\
\hline 6 & - & $45^{2}$ & $5^{6}$ & $37 \mathrm{I}$ & 396 & 440 & 45 & $\begin{array}{l}339 \\
395\end{array}$ & 0 & $\begin{array}{r}25 \\
-\quad \mathbf{I}\end{array}$ \\
\hline 8 & - & 417 & $\begin{array}{l}77 \\
62\end{array}$ & 320 & 340 & $4 \mathrm{I} 4$ & $6 \mathrm{I}$ & 353 & 16 & $\begin{aligned}-1 \\
13\end{aligned}$ \\
\hline & II & $\begin{array}{r}395 \\
540\end{array}$ & & 308 & 333 & 393 & 50 & 343 & 20 & Io \\
\hline $\begin{array}{l}x \\
2\end{array}$ & $\underline{-}$ & $\begin{array}{l}54{ }^{\circ} \\
476\end{array}$ & $\begin{array}{r}100 \\
8 \mathrm{I}\end{array}$ & $\begin{array}{l}394 \\
360\end{array}$ & $\begin{array}{l}440 \\
395\end{array}$ & $\begin{array}{l}522 \\
475\end{array}$ & $\begin{array}{l}81 \\
66\end{array}$ & $\begin{array}{l}44 \mathrm{I} \\
409\end{array}$ & $\begin{array}{l}26 \\
43\end{array}$ & $\begin{array}{r}\mathrm{I} \\
\text { I4 }\end{array}$ \\
\hline 3 & - & 446 & 99 & 340 & 347 & 489 & 79 & 410 & 74 & $\begin{array}{l}14 \\
63\end{array}$ \\
\hline 4 & - & 502 & 68 & 428 & 435 & $53^{8}$ & 54 & 484 & 49 & 49 \\
\hline $\begin{array}{l}5 \\
6\end{array}$ & 二 & 468 & 9I & 384 & 377 & 527 & 73 & 454 & 63 & 77 \\
\hline 7 & - & $\begin{array}{l}463 \\
415\end{array}$ & $\begin{array}{r}72 \\
\text { I08 }\end{array}$ & $\begin{array}{l}408 \\
209\end{array}$ & 391 & 537 & $\begin{array}{l}58 \\
87\end{array}$ & $\begin{array}{l}479 \\
363\end{array}$ & $5^{56}$ & 88 \\
\hline 8 & - & 476 & 67 & $\begin{array}{l}299 \\
366\end{array}$ & $\begin{array}{l}307 \\
409\end{array}$ & $\begin{array}{l}450 \\
468\end{array}$ & $\begin{array}{l}87 \\
54\end{array}$ & $\begin{array}{r}303 \\
4 \mathrm{II} 4\end{array}$ & $\begin{array}{l}6.3 \\
4 I\end{array}$ & $\begin{array}{r}55 \\
5\end{array}$ \\
\hline I & III & 627 & I 08 & 476 & 519 & 636 & 87 & 549 & $6_{4}$ & \\
\hline 2 & - & 560 & 87 & 455 & 473 & 625 & 70 & 555 & 78 & $\begin{array}{l}30 \\
82\end{array}$ \\
\hline 3 & - & 509 & 123 & 414 & 386 & 608 & IOI & 509 & I 16 & 123 \\
\hline 4 & $\cdots$ & 606 & 97 & 493 & 509 & 630 & 78 & $55^{2}$ & 99 & 43 \\
\hline 4 & - & 562 & I 33 & $45^{2}$ & 429 & 644 & 107 & 537 & 106 & 108 \\
\hline 6 & - & $55^{6}$ & 99 & 495 & 457 & 673 & 80 & 593 & $9^{2}$ & ${ }^{1}{ }^{6}$ \\
\hline $\begin{array}{l}7 \\
8\end{array}$ & 一 & $\begin{array}{l}468 \\
55^{2}\end{array}$ & $\begin{array}{r}\mathrm{I} 23 \\
86\end{array}$ & $\begin{array}{l}324 \\
43^{\circ}\end{array}$ & $\begin{array}{l}345 \\
466\end{array}$ & $\begin{array}{l}539 \\
591\end{array}$ & $\begin{array}{l}99 \\
69\end{array}$ & $\begin{array}{l}440 \\
522\end{array}$ & $\begin{array}{r}\operatorname{Ir} 8 \\
86\end{array}$ & $\begin{array}{l}95 \\
56\end{array}$ \\
\hline I & IV & 642 & I 29 & 543 & $5^{\mathrm{I}} 3$ & $73^{8}$ & 103 & 635 & 95 & $\mathrm{I} 22$ \\
\hline 4 & - & 724 & 108 & 610 & 616 & 815 & 87 & 729 & I35 & II3 \\
\hline $\begin{array}{l}5 \\
6\end{array}$ & $\bar{z}$ & & 153 & $55 \mathrm{I}$ & 535 & 787 & 123 & 664 & I 25 & 129 \\
\hline 7 & - & $\begin{array}{l}647 \\
649\end{array}$ & $\begin{array}{l}\text { I29 } \\
\text { I 4 I }\end{array}$ & $\begin{array}{l}5 \mathrm{I} 3 \\
534\end{array}$ & $\begin{array}{l}518 \\
508\end{array}$ & $\begin{array}{l}748 \\
732\end{array}$ & $\mathrm{IO} 3$ & 645 & $\begin{array}{r}165 \\
08\end{array}$ & I 27 \\
\hline 8 & - & 600 & I IO & 504 & 490 & 697 & $\begin{array}{r}\text { II } 4 \\
89\end{array}$ & $\begin{array}{l}618 \\
608\end{array}$ & $\begin{array}{r}9^{8} \\
\quad 39\end{array}$ & $\begin{array}{l}110 \\
118\end{array}$ \\
\hline I & V & 748 & 122 & 660 & 626 & 867 & 98 & 769 & I 37 & $\mathrm{I} 43$ \\
\hline 2 & - & 679 & 94 & 609 & 585 & 793 & 89 & 704 & 160 & 95 \\
\hline $\begin{array}{l}4 \\
5\end{array}$ & E & 792 & 109 & 700 & 683 & 906 & 88 & 818 & I95 & I 35 \\
\hline 6 & 二 & $\begin{array}{l}739 \\
668\end{array}$ & $\begin{array}{l}\text { I35 } \\
\text { I } 77\end{array}$ & $\begin{array}{l}631 \\
584\end{array}$ & 604 & 855 & 109 & 746 & 182 & I42 \\
\hline 7 & - & 708 & I 38 & $\begin{array}{l}304 \\
607\end{array}$ & $\begin{array}{l}55 \mathrm{I} \\
57 \mathrm{O}\end{array}$ & $\begin{array}{l}813 \\
826\end{array}$ & 94 & 719 & I96 & I 68 \\
\hline & - & 746 & 123 & 645 & 623 & 856 & IOI & $\begin{array}{l}715 \\
755\end{array}$ & $\begin{array}{l}\text { I62 } \\
\text { I } 78\end{array}$ & $\begin{array}{l}\text { I } 45 \\
\text { I } 32\end{array}$ \\
\hline $\begin{array}{l}\text { I } \\
2\end{array}$ & VI & $84 \mathrm{I}$ & I37 & 745 & 704 & 1 OI 7 & III & 906 & 192 & 202 \\
\hline 4 & $\overline{-}$ & & IIO & & 679 & $9^{64}$ & 89 & 875 & 205 & 196 \\
\hline 5 & - & $\begin{array}{l}908 \\
814\end{array}$ & $\begin{array}{l}\text { I 4I } \\
\text { I 49 }\end{array}$ & $\begin{array}{l}824 \\
705\end{array}$ & $\begin{array}{l}767 \\
665\end{array}$ & I 077 & $\mathrm{II} 3$ & $9^{64}$ & 215 & I97 \\
\hline 6 & - & 735 & I 14 & $\begin{array}{l}705 \\
637\end{array}$ & $\begin{array}{l}005 \\
621\end{array}$ & 905 & I 20 & 845 & 213 & 180 \\
\hline 7 & - & 814 & I 50 & 709 & 664 & $\begin{array}{l}874 \\
985\end{array}$ & $\begin{array}{r}92 \\
\end{array}$ & 782 & 249 & 161 \\
\hline 8 & - & 896 & r 36 & 776 & 760 & I 035 & $\begin{array}{l}121 \\
109\end{array}$ & $\begin{array}{l}004 \\
926\end{array}$ & $\begin{array}{l}2 \mathrm{I} 2 \\
230\end{array}$ & $\begin{array}{l}200 \\
166\end{array}$ \\
\hline
\end{tabular}


des calories résultant de la combustion des glucides est de I 8I2. Ceci correspond à $\frac{\mathrm{I} 8 \mathrm{I} 2}{3,9}$ de glucose, dont 1'utilisation exige

$$
\frac{\mathrm{I} 8 \mathrm{I} 2}{3,9} \times 0,75=348 \text { litres }
$$

d'oxygène, volume à rapprocher de la donnée expérimentale correspond dante, qui est de 3651 (tableau III).

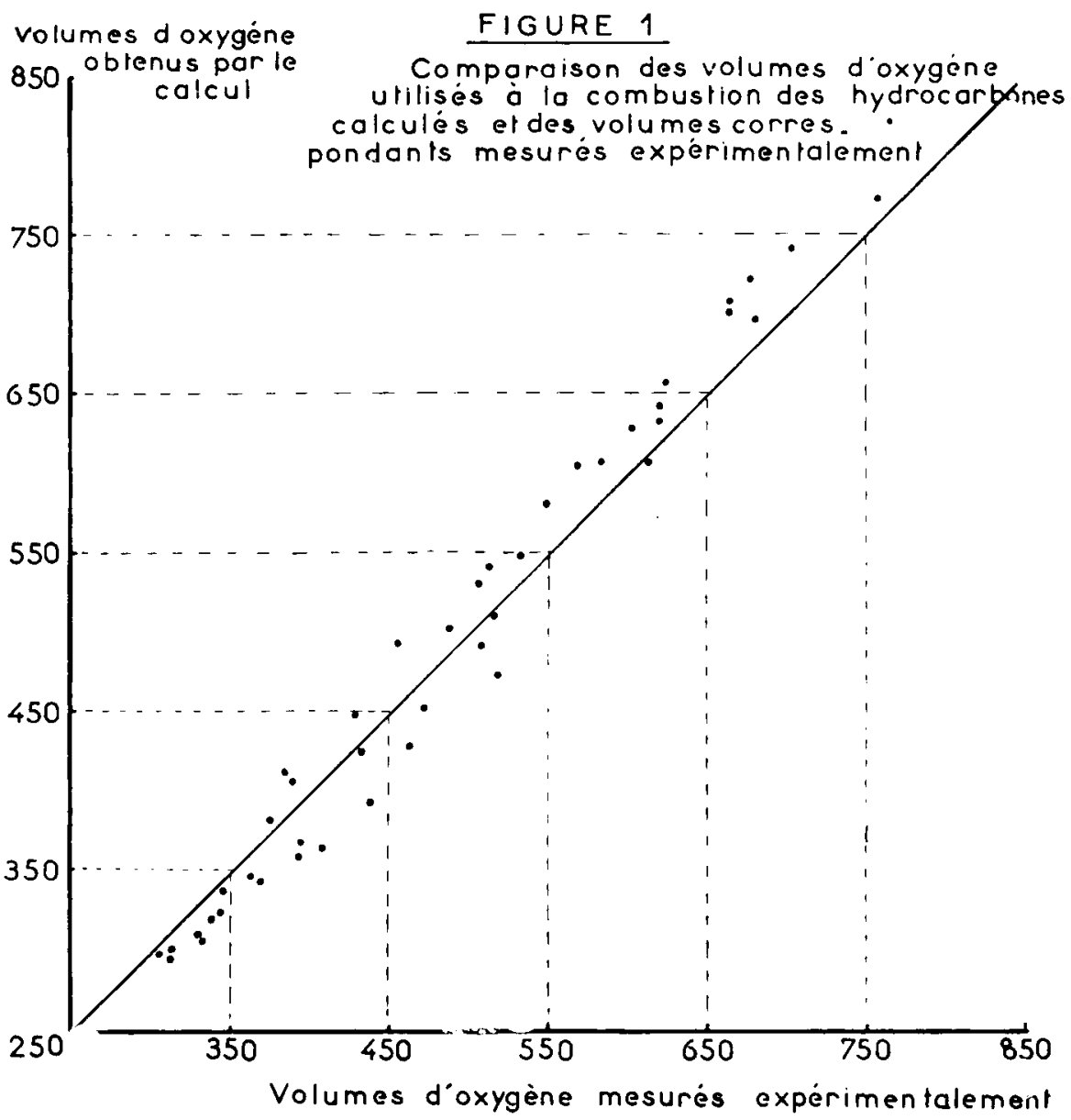

Pour chacune des 44 expériences, de semblables calculs ont été effectués ; leurs résultats figurent dans la colonne 3 du tableau III. Les données ainsi obtenues peuvent être aisément comparées aux résultats expérimentaux correspondants, reproduits dans la colonne $4 \mathrm{du}$ même tableau.

Nous constatons ainsi que les écarts entre les deux séries de données sont tantôt dans un sens, tantôt dans l'autre. Pour mieux comparer ces 
résultats, il est utile de construire un graphique, (Fig. I) obtenu en portant en abscisses les volumes d'oxygène résultant de l'expérimentation directe, et, en ordonnées, les nombres correspondants obtenus par le calcul. Les points représentant chaque couple de données se trouvent ainsi sensiblement rangés autour de la bissectrice de l'angle droit formé par les deux axes, ce qui nous permet de conclure à la similitude des deux séries de mesures.

Si l'on tient compte du fait que les écarts entre les valeurs prises deux à deux ne dépassent jamais Io p. Ioo, on peut admettre qu'elles proviennent des erreurs expérimentales inévitables. L'hypothèse de l'identification à des glucides de la fraction des matières non azotées utilisées à la calorification se trouve donc ainsi vérifiée.

Examinons maintenant les renseignements que nous apportent les mesures expérimentales concernant le gaz carbonique. Pour chaque expérience, il est fait mention, à côté du volume total émis en $24 \mathrm{~h}$ par l'animal, de la fraction de ce gaz correspondant aux matières non azotées. Or, puisque nous savons que les matériaux utilisés à la calorification sont exclusivement des glucides, le volume théorique du gaz carbonique formé au cours de la transformation de ces glucides devrait être identique à celui de l'oxygène (Quotient respiratoire $\frac{\mathrm{CO}^{2}}{\mathrm{O}^{2}}=\mathrm{I}$ ). Mais il existe dans tous les cas - sauf une exception - une différence plus ou moins forte entre le $\mathrm{CO}_{2}$ observé attribué aux glucides et celui de l'oxygène correspondant. La différence entre ces deux volumes provient évidemment du gaz carbonique excédentaire provenant de la transformation d'une certaine partie des glucides en lipides, dont le volume, calculé à l'aide de l'équation d'A. GAUTIER, est deo,6litre degaz carbonique pargramme de matière grasse formée. Or, nous connaissons, pour chaque expérience, la quantité de matière grasse provenant de la transformation des glucides. Il est donc possible, dans ces conditions, de comparer le gaz carbonique excédentaire correspondant à chaque essai à la quantité théorique de ce même gaz obtenu en multipliant le nombre de grammes de graisse formée par le coefficient 0,6 . Les résultats de ces comparaisons figurent dans les colonnes 8 et 9 du tableau III.

Comme nous l'avons fait pour les volumes d'oxygène, nous avons établi un graphique (fig 2) en portant en abscisses les volumes excédentaires de gaz carbonique provenant des données expérimentales, et en ordonnées, les volumes correspondants obtenus par le calcul à partir des matières grasses. Bien que la concordance entre les deux séries soit moins satisfaisante que celle que nous venons précédemment de constater à propos des données relatives à l'oxygène, nous pouvons admettre, en raison des nombreuses causes d'erreurs qui peuvent se produire au cours de semblables observations, que les quotients respiratoires supérieurs à l'unité, qui 
s'observent couramment chez le porc, s'expliquent par le gaz carbonique dégagé au cours de la formation de la graisse aux dépens des glucides.

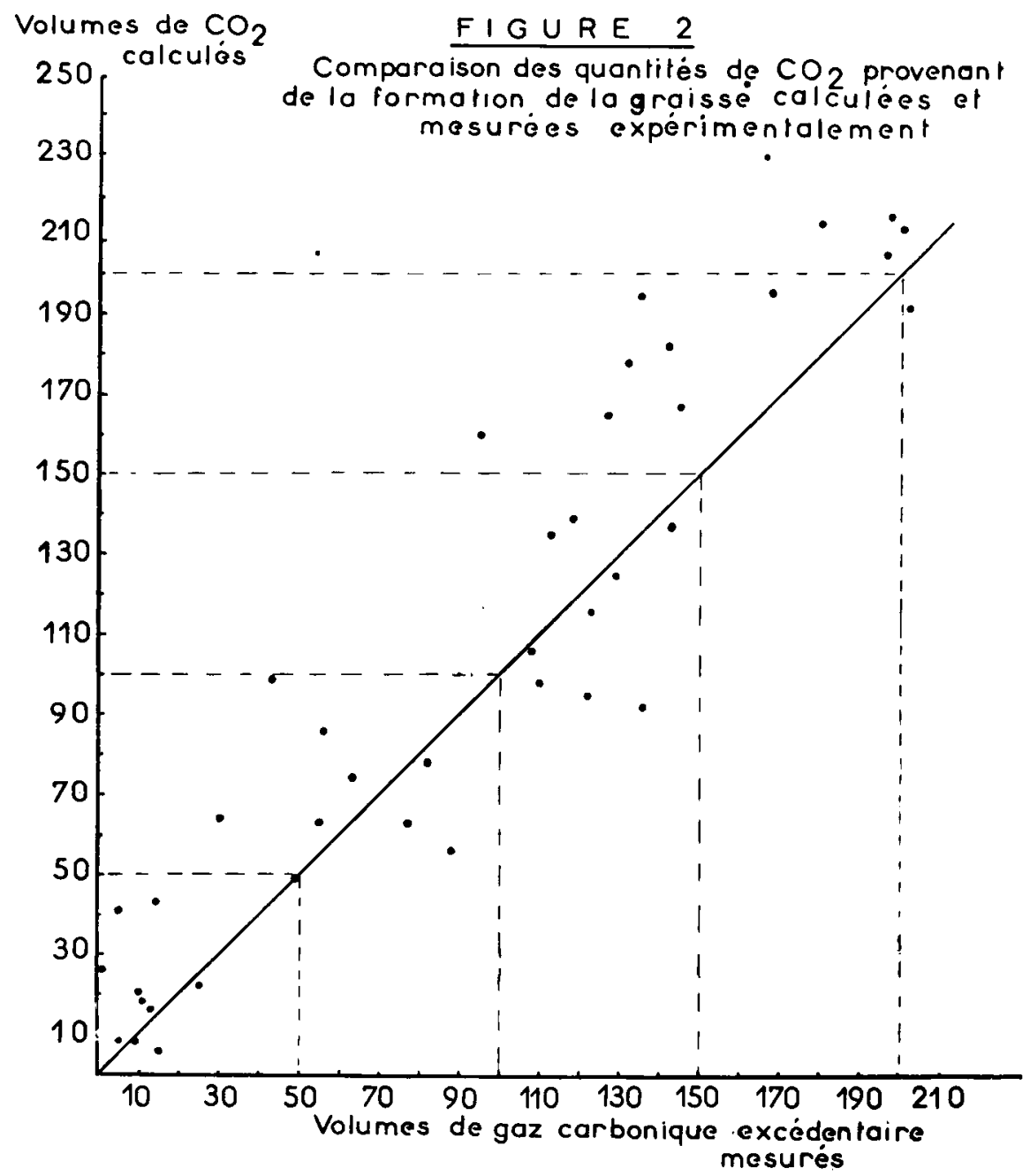

QUATRIÈME, PARTIE

Mise en évidence d'importantes variations individuelles chez des pores soumis au même régime alimentaire.

L'examen détaillé des données analysées montre qu'il existe entre des animaux dont les gains de poids vif sont analogues de considérables différences entre les poids de matières azotées et de matière grasse dont leur corps s'accroit chaque jour. La figure 3, qui indique la composition 
Marières fixées par jour en grs

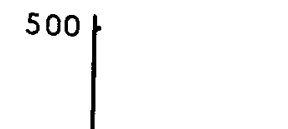

\section{FIGURE 3}

Coinparaison des gains quotidiens de malières grasses el de malíeres azorees de deux porcs $N: 1$ et $N^{0} 6$

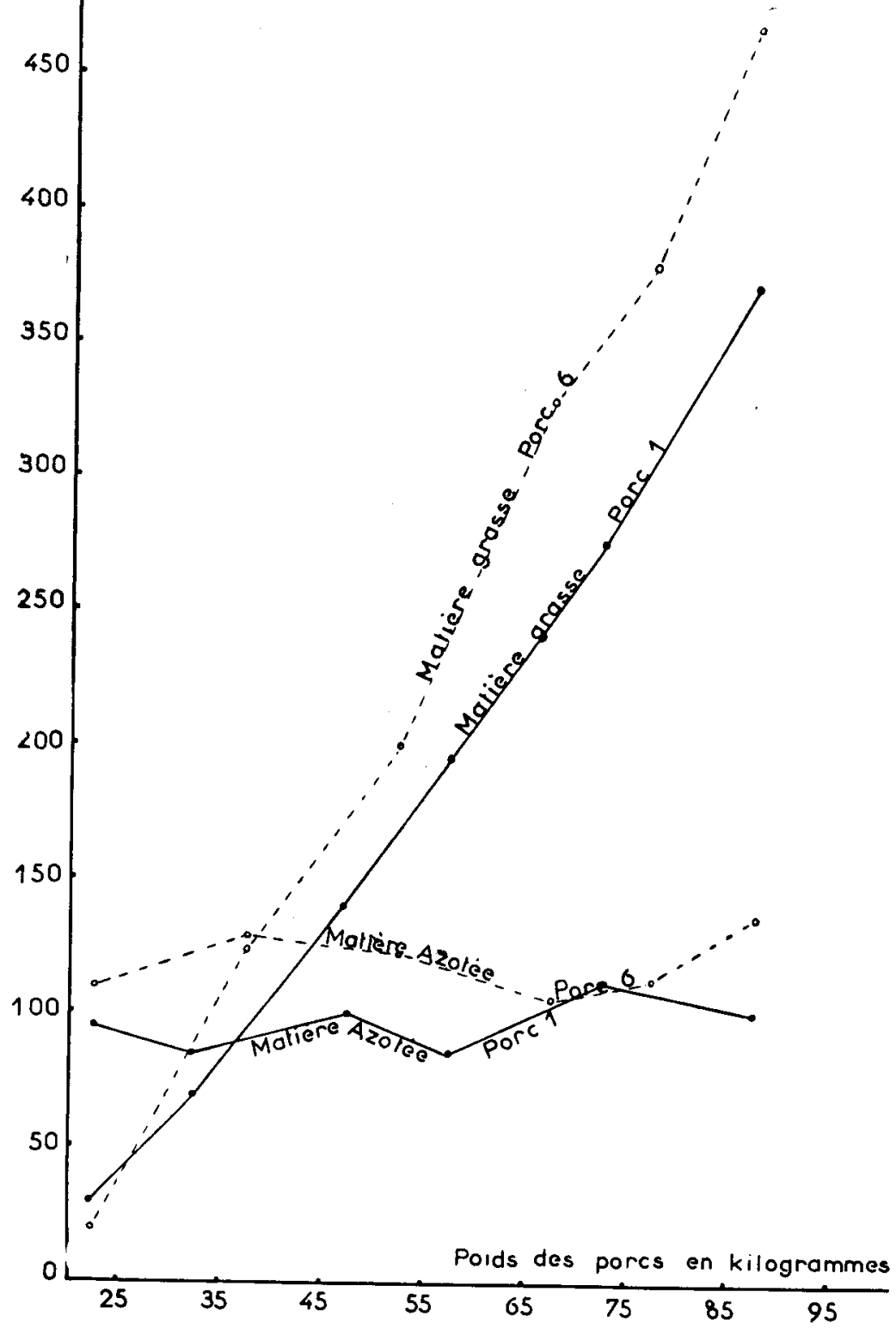

des gains quotidiens des porcs I et 6 , en fonction de leur poids vif, montre nettement que, pour des accroissements de poids sensiblement identiques, 1e premier a toujours fixé moins de matériaux organiques que le second. 
Au cours de l'expérience, les gains quotidiens des deux porcs ont été respectivement de $590 \mathrm{~g}$, pour le $\mathrm{n}^{0} \mathrm{I}$, et de $607 \mathrm{~g}$, pour le $\mathrm{n}^{0} 2$. Les compositions des gains de poids vif entre 20 et $90 \mathrm{~kg}$ ont été les suivants.

\section{TABLEAU IV}

Composition des gains de poids vif moyens, par kilogramme, des porcs $N^{0}$ I et $N^{\circ} 6$

Matières azotées.

Matières grasses..................

Energie potentielle correspondante..

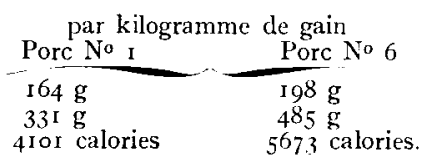

Devant de pareils résultats, il est raisonnable de penser que le porc $n^{0} \mathrm{I}$, moins chargé de graisse que l'autre porc au moment de l'abatage, devait incontestablement mieux convenir aux exigences de la demande actuelle des utilisateurs de viande de porc.

L'examen comparé des données des tableaux II et III semble indiquer que, dans chaque série, les porcs qui obtiennent les gains quotidiens de matière grasse les plus élevés sont ceux pour lesquels la consommation d'oxygène est la plus faible. Pour obtenir la preuve de cette observation, nous avons fait figurer, sur le tableau suivant (tableau V), pour chacune des 6 séries d'expérience, les gains de matière grasse le plus élevé et le plus faible de la série, avec, en regard, les quantités d'oxygène absorbé correspondantes.

\section{Tableau V}

Comparaison de la quantité de matière grasse fixée par les porcs et de la consommation d'oxygène correspondante.

\begin{tabular}{|c|c|c|c|}
\hline $\begin{array}{l}\text { Numéro } \\
\text { de la série }\end{array}$ & Numéro de l'animal & $\begin{array}{c}\text { Cain quotidien } \\
\text { de matićre grasse } \\
\mathrm{g}\end{array}$ & $\begin{array}{c}\text { Quantité totale } \\
\text { d'oxygène consommée } \\
\text { litres }\end{array}$ \\
\hline I $\ldots \ldots$. & 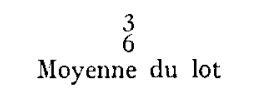 & $\begin{array}{l}6 \mathbf{I} \\
22 \\
48\end{array}$ & $\begin{array}{l}403 \\
45^{2} \\
414\end{array}$ \\
\hline II $\ldots$. & $\begin{array}{l}3 \\
\text { I } \\
\text { Moyenne du lot }\end{array}$ & $\begin{array}{r}157 \\
74 \\
\mathrm{I} 20\end{array}$ & $\begin{array}{l}446 \\
54 \circ \\
473\end{array}$ \\
\hline III $\ldots$ & 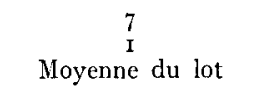 & $\begin{array}{l}234 \\
146 \\
199\end{array}$ & $\begin{array}{l}468 \\
627 \\
555\end{array}$ \\
\hline IV $\ldots \ldots \ldots \ldots$ & 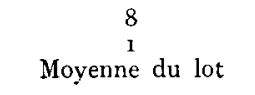 & $\begin{array}{l}28 I \\
199 \\
242\end{array}$ & $\begin{array}{l}600 \\
658 \\
642\end{array}$ \\
\hline $\mathrm{V} \ldots \ldots \ldots \ldots$ & $\begin{array}{c}6 \\
\text { I } \\
\text { Moyenne du lot }\end{array}$ & $\begin{array}{l}381 \\
278 \\
345\end{array}$ & $\begin{array}{l}668 \\
748 \\
726\end{array}$ \\
\hline VI $\ldots \ldots \ldots \ldots$ & $\begin{array}{c}\text { I } \\
\text { Moyenne du lot }\end{array}$ & $\begin{array}{l}480 \\
377 \\
4 \mathbf{2 2}\end{array}$ & $\begin{array}{l}735 \\
84 \mathrm{I} \\
828\end{array}$ \\
\hline
\end{tabular}


En corrigeant les résultats obtenus pour tenir compte dans chaque série de l'influence exercée par les différences des poids des animaux sur la consommation de poids vif et le gain de matière grasse, il a été possible de calculer le coefficient de corrélation entre la consommation totale d'oxygène et l'enrichissement quotidien de matière grasse. Ce coefficient est de $-0,493$.

Nous pensons qu'il convient d'attribuer à cette observation une importance pratique considérable. Les porcs à graisse typiques ont, en effet, une capacité pulmonaire réduite, en raison du faible développement de leur cage thoracique, qui coïncide généralement avec des côtes verticales. L'étude de l'ampleur des mouvements respiratoires chez l'animal vivant et la mesure systématique du volume de la cage thoracique postmortem pourraient à notre avis fournir aux sélectionneurs qui recherchent le porc à viande de très utiles renseignements.

\section{CINQUIÈME PAR'TIF,}

\section{Influence de la formation de la graisse sur le coefficient permettant de calculer l'ensemble des pertes de chaleur provenant des actions dynamiques spécifiques engendrées par la consommation des aliments.}

Dans un précédent travail, nous avons montré que la consommation des aliments entraînait un important accroissement de la dépense énergétique, suivie d'une période de rémission d'autant plus accentuée que la consommation de matière sèche et la quanti 'é d'azote digéré dépassant les besoins de l'organisme sont plus faibles. Si l'on désigne l'énergie métabolisable par M, le besoin d'énergie d'entretien par E, l'énergie potentielle du gain quotidien de matières organiques par $P$, et la matière sèche par Ms, on a

$$
\mathrm{M}=\mathrm{E}+a \mathrm{Ms}+\mathrm{P}
$$

expression dans laquelle $a$ est un coefficient voisin de l'unité, qui, pour les porcs, a été évalué à 0,85 calories.

Pour chacune des 44 expériences, les données publiées permettent de connaître l'énergie totale dissipée sous forme de chaleur. Le poids de l'animal pendant l'essai permet de calculer la quantité d'énergie $\mathrm{E}$ nécessaire à l'entretien. I a différence entre l'énergie totale perdue M et l'énergie d'entretien, $\mathrm{F}$, correspond à l'ensemble des actions dynamiques spécifiques entraînées par la consommation des aliments. Puisque l'on connaît également la quantité correspondante de matière sèche absorbée, $\mathrm{Ms}$, on peut ainsi calculer dans chaque cas la valeur du coefficient $a$, au moyen de la formule $a=\frac{\mathrm{M}-\mathrm{E}}{\mathrm{Ms}}$ 
Ainsi, pour l'expérience faite avec le porc No $I$, au cours de la série I, les valeurs respectives de $\mathrm{M}$ et de Ms sont $2 \mathrm{I} 23 \mathrm{cal}$. et $843 \mathrm{~g}$. Pour un poids de $26 \mathrm{~kg}$, la valeur de Fi est de I o6o calories. Dans ce cas, la valeur de $a$ est de $\frac{2 \text { I23-I } 060}{843}=$ I,24

Le tableau VI nous indique, pour chaque expérience, les résultats de pareils calculs. I a moyenne générale de la valeur de $a$, pour l'ensemble des essais, est de $0,894 \pm 0,013$.

Si nous éliminons la première série de mesures effectuée sur des sujets qui, par manque d'habitude, devaient être particulièrement turbulents, ce qui contribuait vraisemblablement à accroître leurs dépenses, nous constatons que la moyenne des autres déterminations est de 0,86 , nombre presque identique à celui que nous avons nous-même antérieurement publié.

\section{Tableau VI}

Valeurs du rapport $\frac{\mathrm{M}-\mathrm{E}}{\mathrm{Ms}}$ pour chacune des expériences.

\begin{tabular}{|c|c|c|c|c|c|c|c|}
\hline Série & No du Porc & $\begin{array}{l}\text { Poids } \\
\text { de l'animal }\end{array}$ & de $\frac{\stackrel{\text { Valeur }}{\mathrm{M}-\mathrm{E}}}{\mathrm{Ms}}$ & Série & No du Porc & $\begin{array}{l}\text { Poids } \\
\text { de l'animal }\end{array}$ & de $\frac{\mathrm{M}-\mathrm{E}}{\mathrm{Ms}}$ \\
\hline I .... & \begin{tabular}{|c} 
I \\
2 \\
3 \\
4 \\
5 \\
6 \\
7 \\
8 \\
Moyenne
\end{tabular} & $\begin{array}{l}24,2 \\
26,0 \\
26,3 \\
29,0 \\
28,2 \\
26,9 \\
25,4 \\
24,7\end{array}$ & $\begin{array}{l}1,29 \\
0,87 \\
1,05 \\
1,09 \\
0,99 \\
0,76 \\
1,13 \\
1,00 \\
1,02\end{array}$ & IV .... & $\begin{array}{c}1 \\
4 \\
5 \\
6 \\
7 \\
8 \\
\text { Moyenne }\end{array}$ & $\begin{array}{l}56,5 \\
67,0 \\
64,6 \\
63,5 \\
59,0 \\
62,0\end{array}$ & $\begin{array}{l}1,01 \\
0,92 \\
0,95 \\
0,75 \\
1,00 \\
0,74 \\
0,895\end{array}$ \\
\hline II .... & $\begin{array}{c}\text { I } \\
2 \\
3 \\
4 \\
5 \\
6 \\
7 \\
8 \\
\text { Moyenne }\end{array}$ & $\begin{array}{l}35,5 \\
36,4 \\
36,5 \\
39,5 \\
39,0 \\
38,0 \\
35,5 \\
35,0\end{array}$ & $\begin{array}{l}\mathrm{r}, 13 \\
0,87 \\
0,76 \\
0,98 \\
0,88 \\
0,92 \\
0,70 \\
0,85 \\
0,89\end{array}$ & $V \ldots$ & $\begin{array}{c}\text { I } \\
2 \\
4 \\
5 \\
6 \\
7 \\
8 \\
\text { Moyenue }\end{array}$ & $\begin{array}{l}71,4 \\
73,0 \\
81,6 \\
79,4 \\
75,5 \\
73,5 \\
78,3\end{array}$ & $\begin{array}{l}0,99 \\
0,80 \\
0,87 \\
0,83 \\
0,73 \\
0,87 \\
0,84 \\
0,85\end{array}$ \\
\hline III .... & $\begin{array}{c}\text { r } \\
2 \\
3 \\
5 \\
6 \\
7 \\
8 \\
\text { Moyenne }\end{array}$ & \begin{tabular}{|r}
44,0 \\
48,0 \\
48,6 \\
$5 \mathrm{I}, 0$ \\
$5 \circ, 4$ \\
46,7 \\
47,5 \\
Sans lc No
\end{tabular} & $\begin{array}{l}1,04 \\
0,87 \\
0,74 \\
0,87 \\
0,92 \\
0,53 \\
0,77 \\
0,82 \\
0,87\end{array}$ & VI ... & $\begin{array}{c}\text { I } \\
2 \\
4 \\
5 \\
6 \\
7 \\
8 \\
\text { Hoyenne }\end{array}$ & $\begin{array}{l}87,0 \\
89,0 \\
98,3 \\
96,3 \\
20,6 \\
89,0 \\
96,0\end{array}$ & $\begin{array}{l}0,93 \\
0,83 \\
0,99 \\
0,84 \\
0,6,5 \\
0,87 \\
0,01 \\
0,86\end{array}$ \\
\hline
\end{tabular}

Les très faibles différences entre les moyennes des indices $\frac{\mathrm{M}}{\mathrm{Ms}}$ correspondant aux cinq dernières séries montrent qu'il ne semble pas 
exister de corrélation entre les valeurs de ces indices et la quantité de matière grasse formée chaque jour par les porcs.

Le tableau ci-après (tableau VII) indique en effet que les valeurs des indices demeurent sensiblement les mêmes, pour des dépôts quotidiens de matière grasse comprises entre roo $\mathrm{g}$ et $45^{\circ} \mathrm{g}$ par jour.

\section{TABI,EAU VII}

Relation entre l'indice $\frac{\mathrm{M}-\mathrm{E}}{\mathrm{Ms}}$ et la production quotidienne de matière grasse.

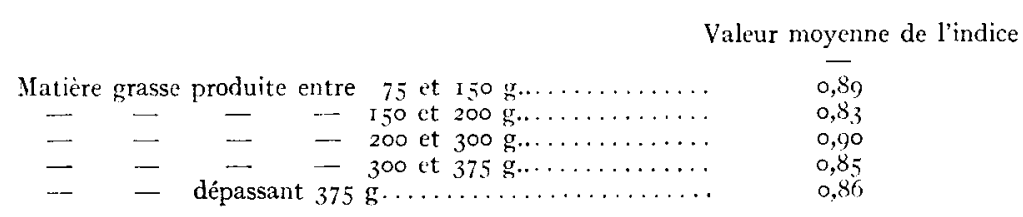

\section{SIXIÈME PARTIE,}

\section{Influence de la formation de la graisse sur le rapport entre le gain de poids vif quotidien et la consommation alimentaire correspon- dante évaluée en unités iourragères.}

Les données numériques contenues dans l'ouvrage de J. I,uDVIGSEN et G. THORBEK permettent aisément de calculer, pour chaque expérience, la valeur énergétique de la ration consommée, évaluée en unités faurragères. La connaissance, dans chaque cas, du poids de l'animal et de son gain quotidien de poids vif permet la détermination de la ration de production par kilogramme de gain. Il suffit, en effet, pour obtenir ce résultat, de déduire du nombre d'unités fourragères représentant la ration totale, celui qui correspond à la ration théorique d'entretien, calculée en fonction du poids vif, et de diviser le résultat par le gain quotidien.

Citons, à titre d'exemple, le cas du porc No I, observé au cours des périodes I et II. I e poids moyen pendant ces 2 périodes était de $28,8 \mathrm{~kg}$ et le gain journalier correspondant, de $433 \mathrm{~g}$, pour une consommation d'aliments représentant $\mathrm{I}, 05$ unité fourragère. I,e besoin d'entretien correspondant est de o $u 43$, ce qui laisse pour la production o $u 62$, soit : $\frac{0,62 \times \text { I } 000}{433}=$ I u 43 par kilogramme de gain.

Nous avons recherché l'existence d'une corrélation entre ces résultats et la quantité journalière de matière grasse accumulée quotidiennement par les porcs au cours de la même période. Comme l'on pouvait s'y attendre, un coefficient de corrélation entre ces deux séries de données s'est montré très élevé $(+0,923)$. I a figure 4 , construite en portant en abscisses les indices de la ration de production par $\mathrm{kg}$ et en ordonnées les quantités 
correspondantes de matière grasse quotidiennement formée, montre avec évidence que l'on peut déduire, avec une certaine approximation, la valeur d'une des deux variables en fonction de l'autre.

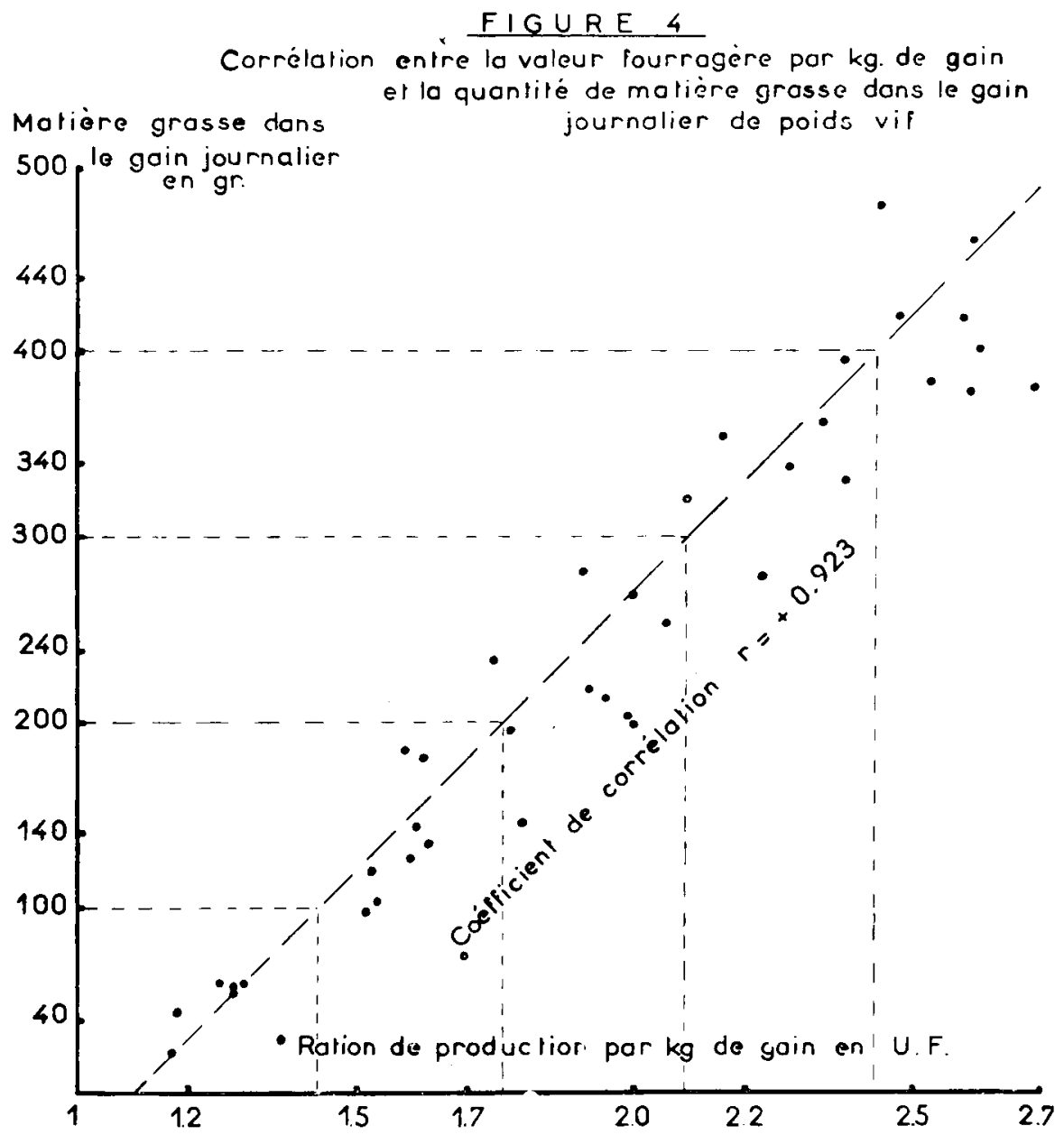

I'observation attentive d'un porc, au cours de sa croissance, pourrait donc ainsi permettre de constater dans quelle mesure cet animal a tendance à former plus ou moins de graisse. Il suffirait, pour cela, de noter exactement la composition de sa ration, ainsi que la valeur alimentaire précise des constituants de cette dernière. Le tracé de la courbe de croissance, obtenu à l'aide de pesées hebdomadaires, pernettrait de calculer pour chaque semaine, en même temps que le gain de poids vif et le poids moyen, l'indice de la ration de production correspondant, duquel il serait possible de déduire la quantité de graisse quotidiennement obtenue. Il n'y 
aurait plus qu'à rapprocher cette quantité de graisse du gain de poids vif observé pour en déduire le pourcentage de matière grasse par rapport à ce même gain.

\section{TABLEAU VIII}

Indice de la ration de production par kilogramme de gain et matières grasses fixées par jour.

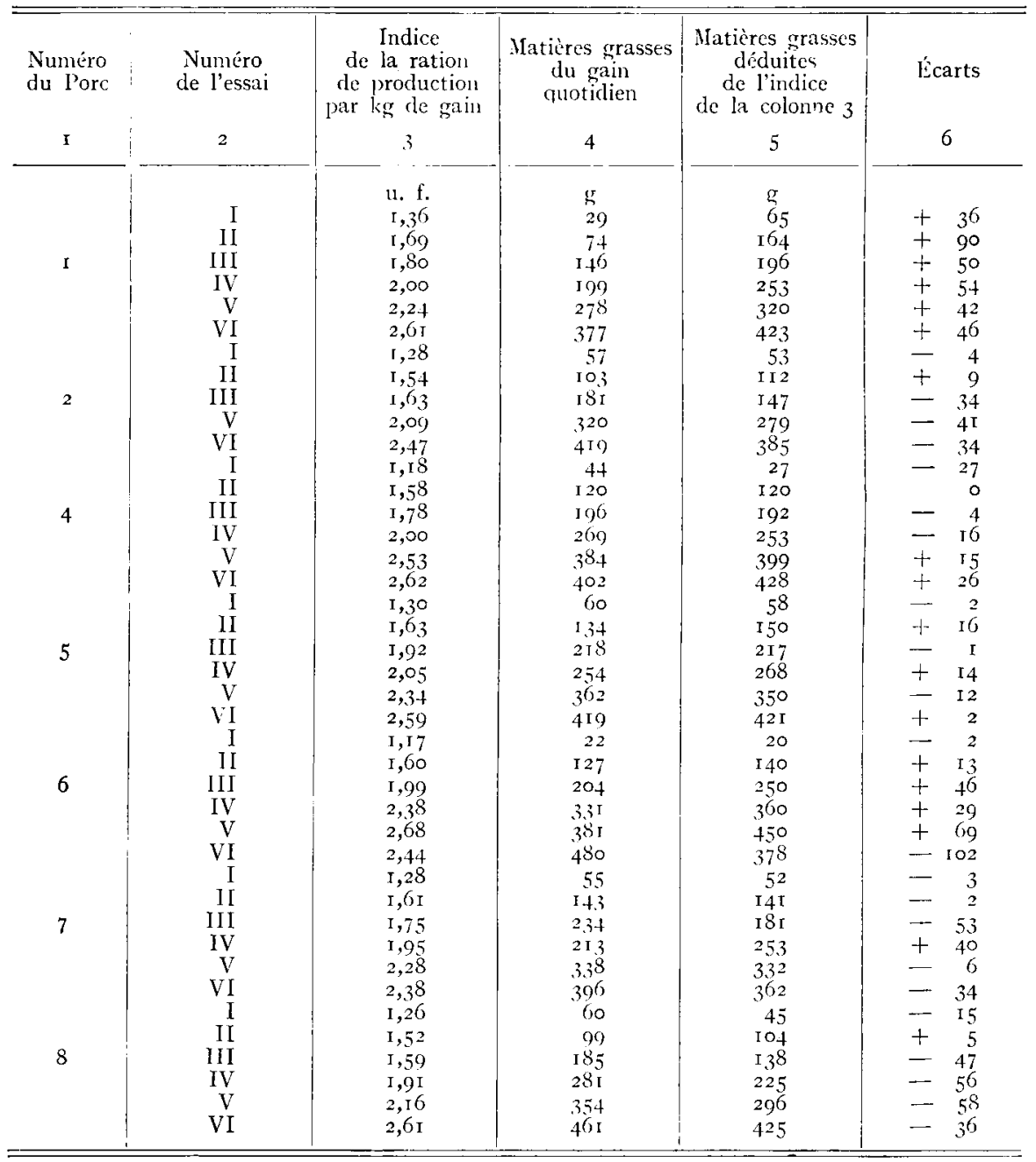

L'étude des données du tableau ci-après (tableau VI) montre que les résultats obtenus par expérience et ceux que l'on peut déduire du besoin de production par $\mathrm{kg}$ de gain ne sont connus qu'à une assez grande approximation. Mais si, au lieu de considérer chaque cas isolément, on consulte la série des données se rapportant à un même porc, il est facile de constater 
que, pour le plus grand nombre de cas, les erreurs en plus ou en moins se compensent. A la condition de multiplier les observations, il semble qu'il y ait beaucoup de chances pour que les indices de la ration de production par kilogramme de gain calculés hebdomadairement pendant toute la durée de l'engraissement d'un porc fournissent une assez bonne mesure de la quantité totale de graisse accumulée en fin d'engraissement dans le corps de cet animal.

Voici, d'après ces renseignements, quelles sont les différences entre les teneurs moyennes en matières grasses par kilogramme de gain, pour l'ensemble de la période d'engraissement, obtenues par l'expérience, d'une part, et par le calcul, d'autre part. On voit ainsi que, dans 5 cas sur 7 , les différences entre les deux mesures sont égales ou inférieures à Io p. IoO

\section{'TABLEAU IX}

Teneurs en matières grasses des gains de poids vif pour l'ensemble de la période d'engraissement, évaluées par kilogramme de gain.

\begin{tabular}{|c|c|c|c|c|c|}
\hline \multirow{2}{*}{$\underset{\substack{\text { Désignation de } \\
\text { l'animal }}}{ }$} & \multirow{2}{*}{$\begin{array}{l}\text { Grain moyen } \\
\text { quotitien } \\
\text { gr. }\end{array}$} & \multicolumn{2}{|c|}{$\begin{array}{l}\text { Matières grasse pour } \\
\text { 100 kg. de grain }\end{array}$} & \multirow{2}{*}{$\begin{array}{l}\text { Différence en } \\
\text { valcur } \\
\text { absolue }\end{array}$} & \multirow{2}{*}{$\%$} \\
\hline & & $\begin{array}{l}\text { Mesurées } \\
\text { expériment. \% }\end{array}$ & $\begin{array}{l}\text { Mesurés par le } \\
\text { calcul } \%\end{array}$ & & \\
\hline 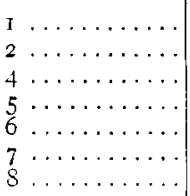 & $\begin{array}{l}588 \\
584 \\
621 \\
604 \\
595 \\
584 \\
635\end{array}$ & $\begin{array}{l}33,1 \\
37,7 \\
32,7 \\
36,6 \\
40,0 \\
39,6 \\
35,4\end{array}$ & $\begin{array}{l}40,3 \\
35,2 \\
3,3,0 \\
37,3 \\
44,0 \\
37,7 \\
3 \mathrm{I}, 5\end{array}$ & $\begin{array}{l}+7,2 \\
+\quad 2,5 \\
+\quad 0,3 \\
+\quad 0,7 \\
+\quad 4,0 \\
-\quad 1,9 \\
-\quad 3,9\end{array}$ & $\begin{array}{r}21,7 \\
6,6 \\
0,9 \\
1,9 \\
10,0 \\
5,0 \\
11,0\end{array}$ \\
\hline
\end{tabular}

\section{CONCLUSION}

De l'ensemble de ce travail, nous pensons pouvoir tirer les conclusions suivantes :

I Lorsque l'on connait la fraction des glucides digestibles utilisés par l'animal pour ses besoins énergétiques, la quantité de matières 'grasses formée quotidiennement est sensiblement égale à la quantité de glucides supplémentaires disponibles, multipliée par 0,37, augmentée de la quantité correspondante des lipides digestibles. L'équation d'Armand GaUTTER, qui rend compte de la transformation du glucose en matière grasse, vérifiée une première fois par ces calculs, se trouve vérifiée une deuxième fois lorsque l'on considère les quantités d'oxygène absorbé et de gaz carbonique rejeté au cours des observations.

$2^{0}$ Il existe une corrélation négative $(-0,49)$ entre la quantité d'oxygène consommée par l'animal en un temps donné et la quantité corres- 
pondante de matière grasse formée. Il résulte de cette observation que les porcs ayant tendance à prođuire exagérément de la graisse ont vraisemblablement une capacité respiratoire inférieure à celle des autres animaux.

$3^{\circ}$ I'énergie dépensée par les porcs en supplément de leur besoin énergétique net d'entretien, qui totalise l'ensemble des actions dynamiques spécifiques entraînées par l'absorption et 1'assimilation des aliments, ainsi que par la désassimilation des nutriments excédentaires, est sensiblement égale, exprimée en grandes calories, au nombre de grammes de matière sèche ingérée multipliée par le coefficient 0,86 .

$4^{\text {o }}$ Il est possible de calculer approximativement la quantité de matière grasse accumulée dans le corps de l'animal au cours de son engraissement, à condition de connaître son gain moyen quotidien de poids vif et ses besoins de production, évalués en unités fourragères par kilogramme de gain, poưr des périodes de durée limitée au cours desquelles la consommation d'aliments et l'accroissement de poids corporel ont été exactement mesurés.

$$
\text { Reçu pour publication le } 9 \text { avril } 1957 .
$$

\section{REFERENCES BIBLIOGRAPHIQUES}

Boussamgault. - Ann. de physique et chimie, $\mathbf{1} 845$.

Dumas, Boussaingault, Payen. - C. R. Ac. des Sciences, I5 février, I843. FINGERLING. - Land. Versuchs Stat., II3, I, I932 ; II4, I, I933 ; II6, I, I933; II8-II9, I, I933; I20-I2I, I, I934.

LEFÈvRE (J.). C Chaleur animale et bioénergétique, IgII, Masson, éd. Paris. LEROY (A. M.). - C.R. Ve Congrès de Zootechnie, Paris, I949.

LEROY (A. M.), FÉVRIER (R.). — Annales agronomiques, 5, I947 ; 4, I949.

LEROY (A. M.), ZELTER (Z.). - Annales de Zootechnie, 1, 6I-77, I952.

LEROY (A. M.). - Annales de Zootechnie, 335-370, 4, I954.

LUdVIGSEN (J.), THORBEK (G.). - 283 Beretning fra forsogslaboratoriet, I955. MolngaARD. - Wissench. A bhand. des deut. Akademie der Lantw. Wissenschaft $z u$ Berlin, 5, 2, I954.

Mussi, et Strohmer. - - Sitzungsber. Berliner Akademie d. Wissenschaft, r893. Richet, Hanriot. - C. R. Ac. Sciences, CXIV, $37 \mathrm{I}$.

RUBNER. - Zeitsch. für Bialogie, I888.

SOXHLEt. - Zeitsch. des Land. Ver. in Bayern, I88r.

Tscherwinsky. - Land. Vers. Stationen, I884.

Vort-Lemmann. - Setzungber bayer. Ak. d. Wissenschajt, I885. 